\title{
Intensive lowering of blood pressure: Should we SPRINT?
}

\author{
Doreen M. Rabi MD MSc, Raj Padwal MD MSc
}

$\mathrm{T}$ he publication of the Systolic Blood Pressure Intervention Trial (SPRINT) challenged the conventional approach to hypertension care. ${ }^{1}$ SPRINT compared intensive lowering of systolic blood pressure (BP) (systolic BP target $\leq 120 \mathrm{~mm} \mathrm{Hg}$ ) with standard treatment (systolic BP target $\leq 140 \mathrm{~mm} \mathrm{Hg}$ ) in 9361 participants aged 50 years and older who had a high risk for cardiovascular disease but no diabetes, and baseline BP levels of 130-180 mm Hg. Eligible participants had either clinical or subclinical cardiovascular disease (excluding stroke and heart failure), a 10-year Framingham Risk Score of $15 \%$ or greater, age 75 years or greater, or chronic kidney disease (estimated glomerular filtration rate $20-60 \mathrm{~mL} / \mathrm{min} / 1.73 \mathrm{~m}^{2}$ ). After 3.3 years, the trial was stopped early. The mean achieved systolic BP level was $122 \mathrm{~mm} \mathrm{Hg}$ in the intensive arm and $135 \mathrm{~mm} \mathrm{Hg}$ in the standard arm. In participants assigned to intensive BP lowering, major adverse cardiovascular events were reduced by $25 \%$ (5.2\% v. $6.8 \%$; hazard ratio [HR] $0.75,95 \%$ confidence interval [CI] 0.64 0.89 ; number needed to treat [NNT] of 62 over $3 \mathrm{yr}$ ) and all-cause mortality was reduced by $27 \%$ (3.3\% v. $4.5 \%$; HR $0.73,95 \%$ CI $0.60-0.90$; NNT of 90 over 3 yr). Notably, no significant reductions were seen in stroke and myocardial infarction.

The results of SPRINT are consistent with the broader literature. A large meta-analysis of 123 placebo-controlled and treat-to-target trials enrolling more than 613000 participants found that lowering systolic BP by $10 \mathrm{~mm} \mathrm{Hg}$ reduces the incidence of major cardiovascular events (HR 0.80, 95\% CI 0.77-0.83), coronary heart disease (HR $0.83,95 \%$ CI $0.78-0.88$ ), stroke (HR 0.73, 95\% CI 0.68-0.77), heart failure (HR $0.72,95 \%$ CI $0.67-0.78$ ), and all-cause mortality (HR 0.87, 95\% CI 0.84-0.91). Importantly, risk reductions are similar when stratified by baseline systolic BP (in quintiles from $<130$ to $\geq 160 \mathrm{~mm} \mathrm{Hg}$ ). ${ }^{2}$ A second meta-analysis focusing only on treat-to-target trials involving patients at high cardiovascular risk (19 trials; nearly 45000 participants) also reported consistent findings for major cardiovascular events, myocardial infarction and stroke. ${ }^{3}$
Based on these data, we believe that intensive lowering of systolic BP should be strongly considered for implementation in general clinical practice. But which patients would benefit, and how should it be done? Four key elements of SPRINT must be emphasized or we cannot hope to realize its benefits in practice.

First, SPRINT enrolled participants at high risk for cardiovascular disease, but the SPRINT definition of high risk is not entirely inclusive (e.g., it excludes patients with diabetes, stroke and heart failure). Implementation in this defined subset of patients only would be prudent, and the criteria for assigning high-risk status need to be clearly delineated..$^{4,5}$

Second, BP measurement in SPRINT consisted of an average of three readings using an automated office BP (AOBP) device following a five-minute rest period. When BP is measured in the office setting, contemporary Canadian hypertension guidelines strongly endorse the use of multiple readings taken using electronic devices (specifically, AOBP devices) while the patient is unattended to minimize the white-coat effect. ${ }^{6,7}$ Further, mean AOBP measurements read 5-10 $\mathrm{mm} \mathrm{Hg}$ lower than routine manual BP measurements. ${ }^{7}$ Practitioners who continue to use manual office measurements risk excessive BP lowering.

Third, SPRINT used protocolized care algorithms emphasizing long-acting agents (especially renin-angiotensin system blockers, thiazide-like diuretics and calcium channel blockers) used in combination. This approach facilitated BP control by limiting therapeutic inertia and ensuring timely medication titration in response to elevated systolic BP levels, and may explain in part why SPRINT investigators were able to lower BP intensively using an average of 2.8 medications. Additionally,

\section{KEY POINTS}

- Intensive lowering of blood pressure (BP) (targeting a systolic BP level of $120 \mathrm{~mm} \mathrm{Hg}$ ) should be strongly considered for selected patients who are at high risk for cardiovascular disease.

- Accurate BP measurement using automated devices and frequent follow-up are necessary to safely achieve intensive targets for systolic BP.

- Shared decision-making, individualized assessment of the benefit-risk profile and the feasibility of close follow-up will need to guide use of this approach for individual patients.
Competing interests: Raj Padwal reports grants from Novo Nordisk and Valencia Technologies, and personal fees from Merck, Servier, Amgen and ProMetic.

This article has been peer reviewed.

Correspondence to: $\mathrm{Ra}$ Padwal, rpadwal@ualberta. ca,rpadwal@yahoo.com

CMAJ 2016. DOI:10.1503 /cmaj.160147 
adherent patients were preselected for inclusion in the study, and patients with resistant hypertension were likely underrepresented.

Fourth, residents of nursing homes and participants with a standing BP of less than $110 \mathrm{~mm} \mathrm{Hg}$, dementia and life expectancy less than three years were excluded.

It is noteworthy that clinically important adverse effects were often seen with intensive therapy. Although the overall rate of adverse events was similar between groups (1793 events [38.3\%] with intensive treatment v. 1736 events [37.1\%] with standard treatment), those receiving intensive treatment had significantly more hypotension ( $2.4 \%$ v. $1.4 \%)$, syncope ( $2.3 \%$ v. $1.7 \%)$, electrolyte abnormalities (3.1\% v. $2.3 \%)$ and acute kidney injury ( $4.2 \%$ v. $2.5 \%)$. These elements underscore the critical importance of appropriate patient selection for intensive BP lowering and that achieving lower systolic targets will involve commensurately intensive clinical and laboratory monitoring to ensure patient safety.

Given the findings of the SPRINT trial and the recent meta-analyses summarized above, targeting a BP of less than $120 \mathrm{~mm} \mathrm{Hg}$ in high-risk patients would seem sensible. However, BP treatment thresholds and targets in patients aged 80 years and older require special consideration. Currently, initiation of pharmacotherapy is recommended if systolic BP is $160 \mathrm{~mm} \mathrm{Hg}$ or greater, with a treatment target of less than $150 \mathrm{~mm} \mathrm{Hg} .{ }^{8}$ Participants aged 75 and older formed a prespecified subgroup of SPRINT; intensive reduction of systolic BP substantially reduced major cardiovascular events in this subgroup (7.7\% v. $10.9 \%$; HR $0.67,95 \%$ CI 0.51-0.86; NNT of 31). The current threshold of $160 \mathrm{~mm} \mathrm{Hg}$ and target of $150 \mathrm{~mm} \mathrm{Hg}$ will need re-examining.

SPRINT does not inform on whether intensive BP lowering is warranted in patients with diabetes, because these individuals were excluded. However, the study's results do provide some insight into why the Action to Control Cardiovascular Risk in Diabetes (ACCORD) trial, which examined intensive BP lowering in patients with diabetes, showed no benefit. ${ }^{9}$ The ACCORD trial was likely underpowered given that it had the same event rate in the standard treatment arm as SPRINT (2.1\%) yet only half the number of participants (4733). Furthermore, the ACCORD trial used a complex factorial design, and interaction between these factorial arms has been demonstrated. ${ }^{10}$

SPRINT also excluded participants with a history of stroke, partly because the European Society of Hypertension (ESH) and the Chinese Hypertension League (CHL) are currently conducting the Stroke in Hypertension Optimal Treatment Trial (ESH-CHL-SHOT). ${ }^{11}$ The trial is enrolling 7500 patients aged 65 and older with a history of prior stroke or transient ischemic attack. The patients are being randomly assigned to three different BP targets $(<145$ to $135 \mathrm{~mm} \mathrm{Hg} \mathrm{v} .<135$ to $125 \mathrm{~mm} \mathrm{Hg}$ v. $<125 \mathrm{~mm} \mathrm{Hg}$ ). Recurrent stroke is the primary end point; results are expected in 2018.

We anticipate some reasonable resistance to the widespread adoption of this approach on the part of both practitioners and patients. More detailed information on adverse effects and additional findings from subgroup analyses as well as cost-effectiveness analyses are needed. Ultimately, shared decision-making, individualized assessment of the benefit-risk profile and the feasibility of close follow-up will need to guide use of this approach for individual patients.

\section{References}

1. Wright JT Jr, Williamson JD, Whelton PK, et al.; SPRINT Research Group. A randomized trial of intensive versus standard blood-pressure control. N Engl J Med 2015;373:2103-16.

2. Ettehad D, Emdin CA, Kiran A, et al. Blood pressure lowering for prevention of cardiovascular disease and death: a systematic review and meta-analysis. Lancet 2016;387:957-67.

3. Xie X, Atkins E, Lv J, et al. Effects of intensive blood pressure lowering on cardiovascular and renal outcomes: updated systematic review and meta-analysis. Lancet 2016;387:435-43.

4. Sundström J, Arima H, Woodward M, et al.; Blood Pressure Lowering Treatment Trialists' Collaboration. Blood pressurelowering treatment based on cardiovascular risk: a meta-analysis of individual patient data. Lancet 2014;384:591-8.

5. Karmali KN, Ning H, Goff DC, et al. Identifying individuals at risk for cardiovascular events across the spectrum of blood pressure levels. J Am Heart Assoc 2015;4:e002126.

6. Daskalopoulou SS, Rabi DM, Zarnke KB, et al. The 2015 Canadian Hypertension Education Program recommendations for blood pressure measurement, diagnosis, assessment of risk, prevention, and treatment of hypertension. Can J Cardiol 2015;31:549-68.

7. Cloutier L, Daskalopoulou SS, Padwal RS, et al. A new algorithm for the diagnosis of hypertension in Canada. Can J Cardiol 2015;31:620-30.

8. Bulpitt CJ, Beckett NS, Peters R, et al. Blood pressure control in the Hypertension in the Very Elderly Trial (HYVET). J Hum Hypertens 2012;26:157-63.

9. Cushman WC, Evans GW, Byington RP, et al.; ACCORD Study Group. Effects of intensive blood-pressure control in type 2 diabetes mellitus. N Engl J Med 2010;362:1575-85.

10. Margolis KL, O'Connor PJ, Morgan TM, et al. Outcomes of combined cardiovascular risk factor management strategies in type 2 diabetes: the ACCORD randomized trial. Diabetes Care 2014; 37:1721-8.

11. Zanchetti A, Liu L, Mancia G, et al.; ESH-CHL-SHOT trial investigators. Continuation of the ESH-CHL-SHOT trial after publication of the SPRINT: rationale for further study on blood pressure targets of antihypertensive treatment after stroke. J Hypertens 2016; 34:393-6.

Affiliations: O'Brien Institute for Public Health (Rabi); Cumming School of Medicine (Rabi), University of Calgary, Calgary, Alta.; Department of Medicine (Padwal), University of Alberta; Alberta Diabetes Institute (Padwal), Edmonton, Alta.

Contributors: Doreen Rabi and Raj Padwal co-conceived this commentary. Doreen Rabi led the writing with iterative input from Raj Padwal. Both authors gave final approval of the version to be published and agreed to act as guarantors of the work.

Funding: Doreen Rabi is supported by a Population Health Investigator award from Alberta Innovates - Health Solutions, the Canadian Institutes of Health Research, the Natural Sciences and Engineering Research Council of Canada, and the Heart and Stroke Foundation of Canada. Raj Padwal is supported by the Canadian Institutes of Health Research, Alberta Innovates Health Solutions, and the University Hospital Foundation. 